paragraphs devoted to distribution, behaviour, enemies, reproductive methods, and so on. To the mind of a systematic zoologist this is undoubtedly preferable to taking these topies in turn and describing the parts they play in the lives of the different taxa; and it may act as a welcome deterrent to a growing practice which threatens to undermine the scientific structure of invertebrate zoology. One can perceive in every one of Cloudsley-Thompson's chapters the clear mind of the professional, expressing its thoughts in lucid, unambiguous language, and making attractive reading from what might seem to be unpromising material. This is no mean achievement.

The illustrations are adequate if not over-plentiful, and the text is always likely to be lightened by reference to some myth or legend that has grown round the really remarkable animals with which the book is concerned. This is wholly legitimate zoology, for it reminds us that the earliest civilized races were far more personally concerned with the irritations of small animals like centipedes and scorpions than they were with the more spectacular lions, elephants or eagles.

There are additions to the bibliographies that close each chapter, bringing these up to date, but corresponding additions to the text are in general limited to a few lines or a paragraph in the appropriate places. 'This seems regrettable. Invertebrate zoology has not stood still during the past decade, and the author has been responsible for a great share in its advances. There may have been reasons for not expanding the book much beyond its former limits, and, in any case, it is very welcome.

THEODORE SAVORY

\section{STUDIES OF BEES}

\section{Sorry, for copyright reasons some images on this page may not be available online}

Metamorphosis of a worker honeybee. One of many photographs by Stephen Dalton in Honeybees from Close Up by Arthur M. Dines (Cassell: London, March 1969, 35s).

\section{SLIME MOULDS}

\section{Biology of the Myxomycetes}

By William D. Gray and Constantine J. Alexopoulos. Pp. viii +288 . (Ronald Press: New York, 1968.) n.p.

Here is a book that, while not contributing much in the way of new ideas, provides a very convenient and useful source of information, much of which has been difficult to find by an independent search of the literature. It is a large and comprehensive review of the biology of the Myxomycetes, and includes, up to 1967, ultrastructural, biochemical and physiological material.

Students of the Mycetozoa generally are rather rare, their publications being outweighed by those of workers using organisms in the group as research materials in investigations of different biological relevance, the stress being on a particular aspect only of their material. such work, however, has contributed to the general understanding of these creatures, and has also drawn them within the view of a wider audience. The Acrasiales have become better known from their morphogenetic interest, and are now better appreciated because of Bonner's book (The Cellular Slime Molds, second edition, 1967). The Myxomycetes are the only other order of the Mycetozoa to have received any widespread attention. This has been chiefly because of their remarkable motility and their dramatic cytoplasmic streaming. These aspects, which have intrigued generations of observers, are still of considerable interest and relevance for biology. Their nuclear behaviour is another feature of topical concern. For workers dealing primarily with such aspects, and who have less interest in the organisms themselves, it has commonly been difficult to trace the relevant literature. One of the functions of this book, and which it performs excellently, is to assemble this literature in a single source. Additionally, it should enlarge the appreciation of biologists for the Myxomycetes.

The chapters of the book follow the course of the life cycle from spore germination through to sporulation, dealing with morphology and physiology side by side where appropriate, although some chapters (for example, "Sporulation") are chiefly physiological, while others (for example, "The Sporophore") are chiefly morphological. The book makes an attempt at critical assessments. though the extent to which this is done is variable, and I got the impression that some whole sections were written chiefly by one or the other author, which may account for the patchiness. The morphological accounts are very well done. So is the chapter on plasmodial locomotion and protoplasmic streaming. These last topics are difficult to review critically, and the appraisal given is good. Other physiological aspects, particularly chemical physiology, seem to me to be much weaker. I was most unsatisfied by the chapter on sporulation, despite the fact that one of the authors (Gray) is known for work on light and sporulation. This section is diffuse and uncritical. Some data are mentioned several times on different pages without their significance being made clear. Table 16 has no value in itself or in conjunction with the text. 'The description of wavelength effects has no discussion of the probability of phytochrome action. There is speculation regarding the possible photoreceptor without an action spectrum being known. There is a failure to distinguish between results obtained from axenic cultures and from those that included other organisms (even though the book elsewhere warns against this failing). Some of the conflicting patterns and nonsense data in this chapter might derive from this point.

A very useful feature of the book is the chapter on laboratory culture. It must be discouraging to myxomycetologists that the ubiquitous Physarum polycephalum attracts such disproportionate attention. With better publicized methods the situation might improve; however, most specios can still be regarded as obligate parasites. 